\title{
Flavor Decomposition for the Proton Helicity Parton Distribution Functions
}

\author{
Constantia Alexandrou $\odot,{ }^{1,2}$ Martha Constantinou, ${ }^{3}$ Kyriakos Hadjiyiannakou, ${ }^{1,2}$ \\ Karl Jansen, ${ }^{4}$ and Floriano Manigrasso (1) ${ }^{1,5,6}$ \\ ${ }^{1}$ Department of Physics, University of Cyprus, P.O. Box 20537, Nicosia 1678, Cyprus \\ ${ }^{2}$ Computation-based Science and Technology Research Center, The Cyprus Institute, 20 Kavafi Street, Nicosia 2121, Cyprus \\ ${ }^{3}$ Department of Physics, Temple University, 1925 N. 12th Street, Philadelphia, Pennsylvania 19122-1801, USA \\ ${ }^{4}$ NIC, DESY, Platanenallee 6, D-15738 Zeuthen, Germany \\ ${ }^{5}$ Institut für Physik, Humboldt-Universität zu Berlin, Newtonstraße 15, 12489 Berlin, Germany \\ ${ }^{6}$ Dipartimento di Fisica, Università di Roma "Tor Vergata”, Via della Ricerca Scientifica 1, 00133 Rome, Italy
}

(Received 5 October 2020; revised 6 November 2020; accepted 12 February 2021; published 12 March 2021)

\begin{abstract}
We present, for the first time, an ab initio calculation of the individual up, down, and strange quark helicity parton distribution functions for the proton. The calculation is performed within the twisted mass clover-improved fermion formulation of lattice QCD. The analysis is performed using one ensemble of dynamical up, down, strange, and charm quarks with a pion mass of $260 \mathrm{MeV}$. The lattice matrix elements are nonperturbatively renormalized and the final results are presented in the $\overline{\mathrm{MS}}$ scheme at a scale of $2 \mathrm{GeV}$. We give results for $\Delta u^{+}(x), \Delta d^{+}(x) \Delta u^{-}(x), \Delta d^{-}(x)$, including disconnected quark loop contributions, as well as for $\Delta s^{+}(x)$ and $\Delta s^{-}(x)$. For the latter we achieve unprecedented precision compared to the phenomenological estimates.
\end{abstract}

DOI: 10.1103/PhysRevLett.126.102003

Introduction.-The theory of the strong interaction, quantum chromodynamics (QCD), describes the structure of hadrons in terms of their constituent quark and gluons (partons), via distribution functions. These are universal quantities and, therefore, can be accessed by a variety of high-energy scattering processes. The cross section of such processes can be factorized into a component calculated in perturbative QCD, and a nonperturbative part expressed in terms of the partonic densities. The latter are the parton distribution functions (PDFs), generalized parton distributions (GPDs), and transverse momentum distribution functions (TMDs), which are necessary for the threedimensional mapping of the hadrons.

At leading order within the parton model, the PDFs have a simple interpretation. The unpolarized PDFs are interpreted as the probability to find an unpolarized parton with a longitudinal momentum fraction $x$ within an unpolarized nucleon. The helicity PDF is the difference between finding quarks with spins aligned and opposite to that of a longitudinally polarized nucleon. The colinear PDFs are completed with the transversity PDFs, which correspond to quarks polarized in the same or opposite direction as a transversely polarized nucleon. High-energy experiments

Published by the American Physical Society under the terms of the Creative Commons Attribution 4.0 International license. Further distribution of this work must maintain attribution to the author(s) and the published article's title, journal citation, and DOI. Funded by SCOAP ${ }^{3}$. (see, e.g., Refs. [1-3]) provide a wealth of measurements that are collectively analyzed using phenomenological fits. Based on the available experimental data, the most wellstudied PDFs are the unpolarized, followed by the helicity with an order of magnitude less experimental data, namely, a few hundred datasets $[4,5]$. The transversity PDFs are even less known [6]. The accessible kinematic region is more limited for the helicity and transversity PDFs as compared to the unpolarized PDFs, and therefore, the reconstruction of PDFs uses input from models. Therefore, the extraction of the helicity and transversity PDFs are, to some extent, driven by the fit functions (see, e.g., Ref. [6]). The dependence on the analysis procedure is evidenced by the tension among some of the global analyses [5,7-9].

The focus of this work is the helicity PDFs, which are typically accessed experimentally in deep-inelastic scattering (DIS), semi-inclusive DIS, Drell-Yan, and protonproton scattering processes. Currently, the global analyses use next-to-leading order (NLO) corrections in perturbative QCD (NNPDF POL $_{1.1}$, DSSV14, JAM17) [5,7-9]. In these analyses, the up and down contributions, $\Delta u(x), \Delta d(x)$ are better constrained in the valence sector, with $\Delta u(x)$ being more precise. On the other hand, constraining $\Delta s(x)$ is not successful, as the kinematic regions of some of the datasets (e.g., the $W$-boson production data) are not sensitive to the strangeness [5]. The situation improves with the inclusion of kaon production SIDIS data, but it is still unsatisfactory, and influenced by theoretical assumptions. In the recent work 
of the JAM Collaboration [7] inclusive and semi-inclusive data were used, finding the strange polarization to be very small and consistent with zero.

Based on the current status of phenomenological analyses, an extraction of the PDFs from theory is highly desirable. Here, we present the first extraction of the up, down, and strange helicity PDFs for the proton within lattice QCD, the only known $a b$ initio formulation of QCD. We study both the valence and sea quark contributions that allow one to perform a controlled decomposition of the $u$ and $d$ PDFs. To obtain the PDFs, we implement the quasi-PDF method [10], which is based on correlation functions that are calculable on a Euclidean lattice. The matrix elements are between proton states with a finite momentum $\vec{P}=\left(0,0, P_{3}\right)$. The proton is coupled with a nonlocal operator with fermion fields separated by a distance $z$ connected by a Wilson line, in the same spatial direction as $\vec{P}$. Thus, the matrix elements are defined in coordinate space, with $z$ varying from zero up to half the spatial extent of the lattice. To extract physical quantities, a Fourier transform is applied on the matrix elements to obtain the so-called quasi-PDFs in momentum space, $x$. For large values of $P_{3}$, the momentum boost can be interpreted as a Lorentz boost, recovering the light-cone PDF. The difference between quasi-PDFs and light-cone PDFs is $\mathcal{O}\left(\Lambda_{\mathrm{QCD}}^{2} / P_{3}^{2}, m_{N}^{2} / P_{3}^{2}\right)$ and is calculable in continuum perturbation theory within the large momentum effective theory (LaMET) [11]. A successful research program on obtaining the PDFs using the quasi-PDFs method was developed since Ji's proposal, leading to theoretical and numerical advances [12-51]. Recently, an exploratory study appeared on the strange and charm unpolarized PDFs [52] using ensembles with pion mass 310 and $690 \mathrm{MeV}$. However, the work only presents matrix elements in coordinate space. Other methods on extracting the $x$ dependence of distribution functions have been discussed [53-81]. For an extensive review of the lattice calculations using the quasi-PDFs method, as well as other approaches to extract PDFs, see Refs. [82-84].

Lattice implementation.-Based on the quasi-PDFs approach, the light-cone PDFs are obtained by the convolution of quasi-PDFs and the corresponding analytic expression for the matching kernel calculated in continuum perturbation theory. The quasi-PDFs are defined in momentum space as

$$
\tilde{\Delta} q(x, \mu, P)=2 P_{3} \int_{-\infty}^{+\infty} \frac{d z}{4 \pi} e^{-i x P_{3} z} \mathcal{M}^{R}\left(z, P_{3}\right),
$$

and are Fourier transform of hadronic matrix elements

$$
\begin{gathered}
\mathcal{M}^{R}\left(z, P_{3}, \mu\right) \equiv Z(z, \mu) \mathcal{M}\left(z, P_{3}\right), \\
\mathcal{M}\left(z, P_{3}\right) \equiv\left\langle N(P)\left|\bar{\psi}(z) \gamma^{3} \gamma^{5} W(0, z) \psi(0)\right| N(P)\right\rangle .
\end{gathered}
$$

The proton initial and final states, $|N(P)\rangle$, carry the same momentum $P=\left(P_{0}, 0,0, P_{3}\right)$, as the PDFs are obtained in the forward kinematic limit. Here we focus on the helicity PDFs, $\Delta q \equiv g_{1}^{q}(x)$, and therefore use the axial nonlocal operator. The bare matrix elements $\mathcal{M}\left(z, P_{3}\right)$ must be renormalized with an appropriate renormalization function, $Z(z, \mu)$, to remove divergences. We calculate $Z(z, \mu)$ using the RI'-type prescription proposed in Refs. [16,17]:

$$
\left.\frac{Z(z, \mu)}{12 Z_{q}^{-1}(\mu)} \operatorname{Tr}\left\{\mathcal{V}_{\gamma^{3} \gamma^{5}}(p, z)\left[\mathcal{V}_{g_{T}}^{\text {Born }}(p, z)\right]^{-1}\right\}\right|_{p^{2}=\mu_{0}^{2}}=1,
$$

which is applied at each value of $z$ separately. We refer the reader to Ref. [34] for notation. Because of the presence of the Wilson line, extracting the singlet renormalization functions is very challenging, as it involves a disconnected diagram. Here we use the nonsinglet function indicated by $Z(z, \mu)$. We note that the difference between the singlet and nonsinglet renormalization functions is expected to be small, as is the case of the local axial operator [85]. The difference between singlet and nonsinglet arises to two loops in perturbation theory [86], explaining its small value. In addition to the logarithmic divergences and finite renormalization, the definition of Eq. (4) also removes the power-law divergence of the Wilson line. $Z(z, \mu)$ is obtained at an $\mathrm{RI}^{\prime}$ scale $\mu_{0}$. In our analysis, we convert to the $\overline{\mathrm{MS}}$ scheme at a scale $\mu=2 \mathrm{GeV}$. An additional conversion factor is used to bring $Z(z, \mu)$ in the modified $\overline{\mathrm{MS}}$ scheme [34]. Therefore, the scale dependence appears in the renormalized matrix element $\mathcal{M}^{R}\left(z, P_{3}, \mu\right)$. While the matrix elements of local operators mix under renormalization [87], the nonlocal operators under study do not mix in the renormalization process, as discussed in Refs. [32,33,35]. This is because there is no additional nonlocal ultraviolet divergence in the quasi-PDF, an argument that holds to all orders in perturbation theory. However, the mixing occurs at the matching level and should be eliminated. To disentangle the singlet helicity PDFs requires the matrix elements of the gluon helicity PDFs, which is beyond the scope of this work. The nature of the mixing was also discussed earlier in Ref. [21] using the auxiliary field approach.

The most widely used method to obtain the quasi-PDFs is via the discretized Fourier transform of Eq. (1). More recently, alternative reconstruction techniques are being explored $[44,70,81,88,89]$. In this work, we compare the standard Fourier transform, with the Bayes-Gauss-Fourier transform [89], finding agreement within uncertainties. We thus present results using the discretized Fourier transform.

As can be seen in Eq. (1), the quasi-PDFs depend on the nucleon momentum $P_{3}$, which should be finite but large. This dependence is expected to be removed by the matching kernel 


$$
\Delta q(x, \mu)=\int_{-\infty}^{\infty} \frac{d \xi}{|\xi|} C\left(\xi, \frac{\mu}{x P_{3}}\right) \tilde{\Delta} q\left(\frac{x}{\xi}, \mu, P_{3}\right),
$$

which is calculated to a given order in continuum perturbation theory. The matching kernel for the quasiPDFs approach has been extensively studied (see, e.g., Refs. [22-24,45-49]). In this work we employ the one-loop matching kernel in the modified $\overline{\mathrm{MS}}$ scheme, as defined in Ref. [34]. Note that we choose the factorization scale to be the same as the renormalization scale $\mu$. The final step in extracting the light-cone PDFs is the application of the nucleon mass corrections, which have been calculated analytically in Ref. [13].

Numerical methods.-Obtaining $\mathcal{M}\left(z, P_{3}\right)$ is the most computationally demanding part of the calculation, as it contains a nonlocal operator, and must be calculated in the boosted frame. We perform the calculation including, for the first time, connected and quark-disconnected diagrams, for both the light and strange quark. In the light-quark sector, we extract the isovector and isoscalar combinations, which are decomposed into the up and down quark helicity PDFs. The calculation is performed using an ensemble of two light, a strange and a charm quark $\left(N_{f}=2+1+1\right)$ within the twisted mass fermion formulation with clover term, produced by the Extended Twisted Mass Collaboration [90]. The lattice spacing is $a=0.093 \mathrm{fm}$ and the lattice volume is $32^{3} \times 64(L \approx 3 \mathrm{fm})$. The pion mass is about $260 \mathrm{MeV}$ and $m_{\pi} L \approx 4$.

The evaluation of the connected diagram uses the techniques outlined in Ref. [34], including the implementation of the momentum smearing method [91], and five stout smearing steps with parameter $\rho=0.15$, on the Wilson line entering the operator. Both smearing methods contribute to the reduction of the statistical noise. We refer to Ref. [34] for the details. We use a total number of measurements $N_{\text {meas }}=392,1552$, and 6320, for momenta $P_{3}=0.41,0.83$, and $1.24 \mathrm{GeV}$, respectively. The sourcesink separation is $t_{s}=0.94 \mathrm{fm}$ for the lowest momentum and $t_{s}=1.13 \mathrm{fm}$ for the other two.

The evaluation of the quark-disconnected diagrams involves the computation of disconnected quark loops that have to be combined with the nucleon two-point correlators. The quark loop with Wilson line reads

$$
\begin{aligned}
& \mathcal{L}\left(t_{\mathrm{ins}}, z\right) \\
& =\sum_{\vec{x}_{\mathrm{ins}}} \operatorname{Tr}\left[D_{q}^{-1}\left(x_{\mathrm{ins}} ; x_{\mathrm{ins}}+z\right) \gamma^{3} \gamma^{5} W\left(x_{\mathrm{ins}}, x_{\mathrm{ins}}+z\right)\right],
\end{aligned}
$$

where $D_{q}^{-1}\left(x_{\text {ins }} ; x_{\text {ins }}+z\right)$ is the quark propagator, whose endpoints are connected by a Wilson line. To reduce the stochastic noise coming from the low modes [92], we computed the first $N_{e v}=200$ eigenpairs of the squared Dirac twisted-mass operator. From the eigenpairs, the lowmodes contribution to the all-to-all propagator can be exactly reconstructed and the high-modes contribution can then be evaluated with stochastic techniques. In particular, the stochastic evaluation of the disconnected loops is based on well-established techniques developed for local operators, such as hierarchical probing [93]. The latter allows for reduction of the contamination of the offdiagonal terms in the evaluation of the trace of Eq. (6), up to a distance $2^{k}$. This is done using Hadamard vectors as basis vectors for the partitioning of the lattice. Here, the hierarchical probing algorithm has been implemented with $k=3$ in 4 dimensions, leading to 512 Hadamard vectors. In addition, for the stochastic evaluation of the disconnected loops we make use of the one-end trick [94,95] and fully dilute spin and color sub-spaces. We have employed successfully such methods in other studies of disconnected contributions [85,96-98].

Results for the connected and disconnected contributions. - For each value of the proton momentum, $P_{3}=0.41,0.83$, and $1.24 \mathrm{GeV}$, we compute the two-point correlator for 200 source positions to reach a good statistical accuracy. We also take all spatial orientations of $P_{3}$ and $W$, that is, $\pm x, \pm y, \pm z$. Moreover, both in the two-point and disconnected three-point functions we average over the forward and backward contributions. The total number of configurations analyzed is 330 for the two smallest momenta, and 480 for the largest one, bringing the total statistics to 66000 and 96000 , respectively. Momentum smearing is applied for the two largest values $P_{3}=0.83,1.24 \mathrm{GeV}$. The gauge links in the Wilson line entering the disconnected loop of Eq. (6) undergo 10 iterations of stout smearing, with parameter $\rho=0.129$.

To properly take into account the contamination of the excited states occurring at small source-sink separations $t_{s}$, we compute the disconnected three-point correlators at $t_{s}=0.75,0.84,0.94,1.03$, and $1.13 \mathrm{fm}$, and perform a two-state fit analysis, following the procedure described in Ref. [96]. We find that the plateau values converge to one extracted using the two-state fit. For all $z / a$ and $P_{3}$ the plateau value for $t_{s}=1.13 \mathrm{fm}$ is in agreement with the one extracted from the two-state fit. For example for the isoscalar combination at $z / a=0$ and $P_{3}=0.41 \mathrm{GeV}$ we find $\mathcal{M}^{(2 s t)}(z / a=0)=-0.19(5)$ using the two-state fit, while the plateau value is $\mathcal{M}^{\text {(plt) }}(z / a=0)=-0.16(3)$. We will use the results from the plateau method in what follows. In Fig. 1 we show the renormalized matrix elements using $t_{s}=1.13 \mathrm{fm}$ for the disconnected contributions. The real strange matrix element is about half as compared to the disconnected $u+d$. The latter is omitted from Fig. 1 since it shows the same qualitative behavior as the strange matrix elements. For both $u+d$ and strange we clearly obtain a nonzero signal with statistical uncertainties under control. The imaginary part of the disconnected matrix elements is at least 1 order of magnitude smaller than the real part. We note that the increase in the error for $z=8 a$ in the disconnected part of the matrix element is due to using hierarchical probing with length $2^{k}$ and $k=3$. 


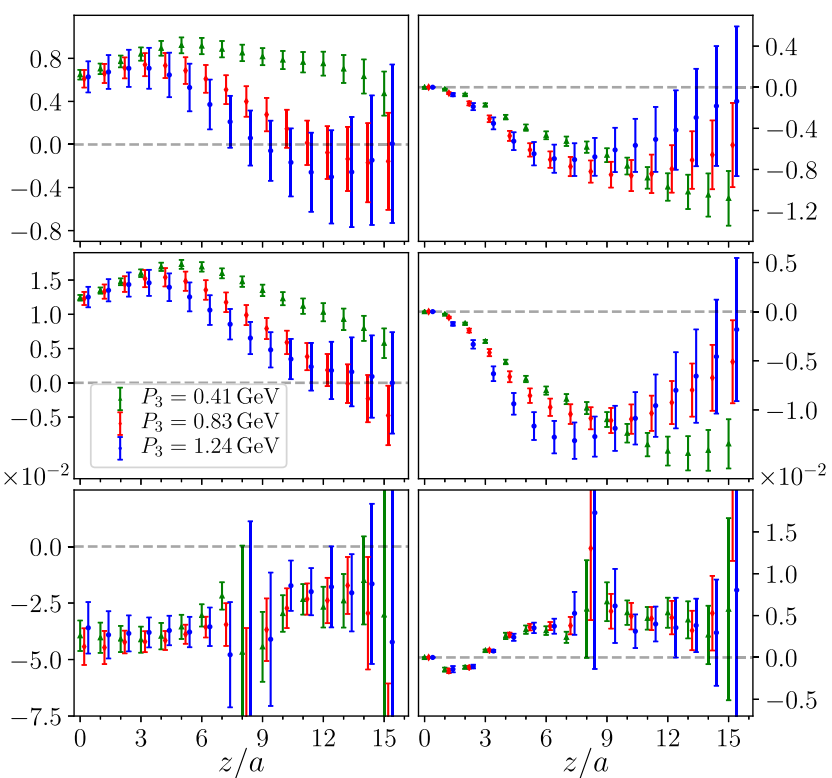

FIG. 1. Real (left) and imaginary (right) part of the renormalized matrix elements of the isoscalar $u+d$ connected (upper panel) and isovector $u-d$ (middle panel) and the strange quark (lower panel).

This is verified by repeating the evaluation of the disconnected diagrams with $k=2$, and confirm that the same behavior occurs at $z=4 a$ and its multiples, reflecting the limitation of the hierarchical probing technique when dealing with large lengths of the Wilson line. In taking the Fourier transform in Eq. (1), we choose the cutoff $z_{\max }$ such that the renormalized matrix element is compatible with zero. Since for the isoscalar and isovector matrix elements this occurs at different values of the Wilson line length $z$, we use different cutoffs $z_{\max }$ for the two quantities. In particular, for the isoscalar case (the sum of connected and disconnected contributions) at $P_{3}=1.24 \mathrm{GeV}$, we use $z_{\max }=7 a$, which is below the hierarchical probing length of $8 a$. While, for the isovector case, we employ $z_{\max } / a=10$.

Two additional important issues need to be addressed in order to extract the PDFs, namely, the dependence of the results on the momentum boost and the accuracy of the discrete Fourier transform. We examine these issues by considering the $x \Delta d^{+}(x) \equiv x(\Delta d+\Delta \bar{d})$ distribution, since the behavior is similar for the other two. To extract the $\Delta d^{+}(x)$ distribution we apply renormalization and matching procedures separately on the isovector, isoscalar, and strange quasi-PDFs. As mentioned above, we neglect the mixing with the gluon helicity PDFs at the matching level.

In Fig. 2 we show the momentum dependence of $x \Delta d^{+}(x)$. We observe that, while when increasing the momentum from 0.41 to $0.83 \mathrm{GeV}$ there is a discrepancy in particular for large values of $x$, when we further increase the momentum to $P_{3}=1.24 \mathrm{GeV}$, the results become

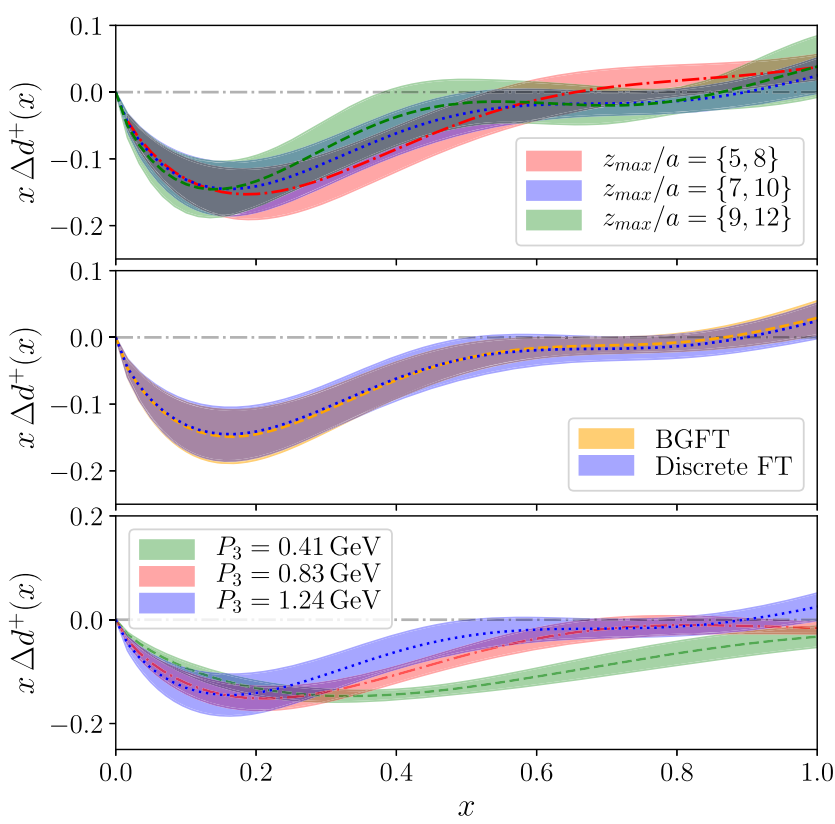

FIG. 2. Dependence on the cutoff $z_{\max }$ of the $\Delta d^{+}$distribution (upper panel) at $P_{3}=1.24 \mathrm{GeV}$. The first(second) number reported between curly brackets indicates the value of $z_{\max }$ adopted with the isoscalar (isovector) matrix element. Comparison between the $\Delta d^{+}$distribution at $P_{3}=1.24 \mathrm{GeV}$ obtained with discrete Fourier transform and with the BGFT technique [89] (middle panel), using the cutoff $\{7,10\}$ in both cases. Momentum dependence of the distribution $\Delta d^{+}$(bottom panel).

compatible. This suggests that convergence has been reached within the limits of our current precision. In Fig. 2 we also show the dependence of the $x \Delta d^{+}(x)$ distribution on the cutoff $z_{\max }$ adopted in the computation of the isoscalar and isovector quasi-PDFs. Despite the fact that when increasing $z_{\max }$, the resulting distribution tends to show more pronounced oscillations, the results for different $z_{\max }$ all agree within uncertainties. In order to estimate the extent of the systematic effect due to the discretization and truncation of the Fourier transform (FT), we employ the Bayes-Gauss-Fourier transform (BGFT) [89]. As can be seen in Fig. 2, the distribution obtained with the BGFT technique is compatible with the standard reconstruction based on the discrete FT.

Flavor decomposition and comparison with phenomenology.-The aim of this work is to obtain the flavor decomposition of the up, down, and strange quark distributions, by combining the total isoscalar and isovector contributions at each $P_{3}$ value. In Fig. 3 we show our final results at $P_{3}=1.24 \mathrm{GeV}$ for $|x| \Delta q^{+}(x) \equiv|x|(\Delta q+\Delta \bar{q})$ and $|x| \Delta q^{-}(x) \equiv|x|(\Delta q-\Delta \bar{q})$, for $q=u, d, s$, and compare with the JAM17 [7] and $\mathrm{NNPDF}_{\mathrm{POL}} 1.1[5,99]$ data. We find that $x \Delta d^{+}(x)$ and $x \Delta s^{+}(x)$ nicely decay to zero at $x=1$. While $x \Delta u^{+}(x)$ is also zero at $x=1$ and in agreement with the JAM17 results for $x \lesssim 0.6$ and with the $\mathrm{NNPDF}_{\mathrm{POL}} 1.1$ data for $x \lesssim 0.5$, it decays slower than the 

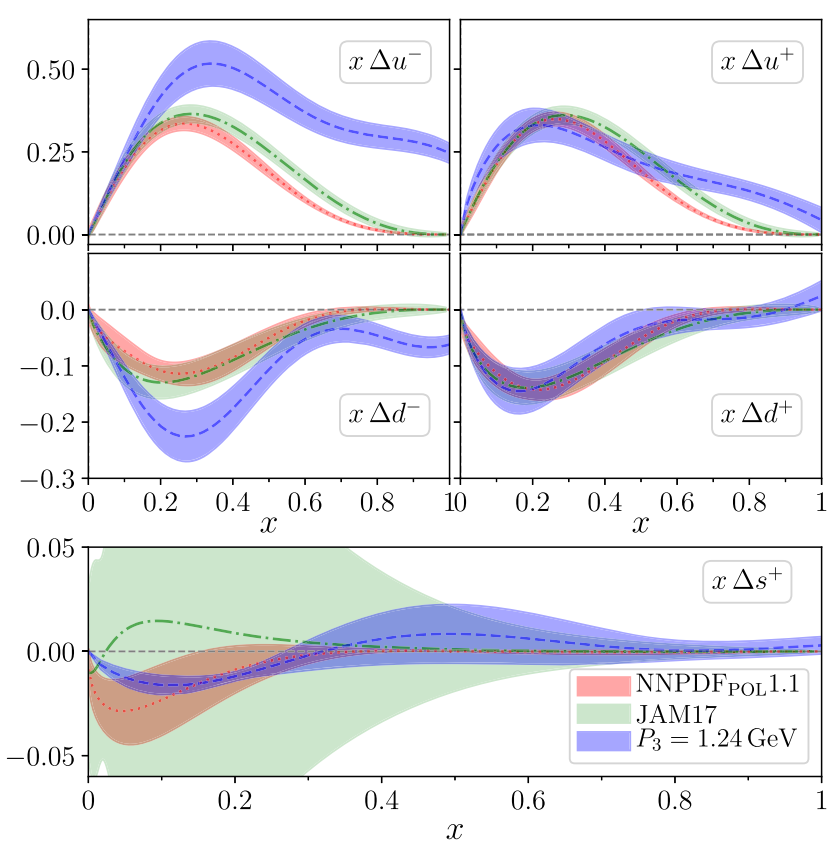

FIG. 3. Comparison of our results on the up (upper), down (middle), and strange (bottom) quark helicity PDFs (blue) in the $\overline{\mathrm{MS}}$ scheme at $2 \mathrm{GeV}$ with the JAM17 [7] (green) and $\mathrm{NNPDF}_{\mathrm{POL}} 1.1[5,99]$ (red) phenomenological datasets.

phenomenological distributions. On the other hand, we find a remarkable agreement for $x \Delta d^{+}(x)$ for the whole $x$ region. Both $x \Delta u^{-}$and $x \Delta d^{-}$distributions are compatible with phenomenology for $x \lesssim 0.2$, but are bigger in magnitude for $x>0.2$, with the former having a larger deviation from zero at $x=0$. Such deviations are expected to be due to lattice artifacts, such as a larger than physical pion mass, and finite lattice spacing effects that are currently under investigation in the isovector channel [100].

Our determination of the strange distribution $x \Delta s^{+}(x)$ is more precise as compared to the one determined from both the JAM17 and the NNPDF POL $_{1.1}$ analyses. We note that for the latter the higher accuracy is achieved by assuming $\mathrm{SU}(3)$ which is not imposed for the former. Moreover, unlike the phenomenological determinations, we do not force the distributions to go to zero at $x=1$. Our results for $x \Delta s^{+}$are compatible for the whole $x$ region with these global analyses. Since the imaginary part of the strange quark renormalized matrix elements is almost zero as shown in Fig. 1, the distribution $\Delta s^{-}(x)$ is zero and thus not shown here. We find that our results confirm a nonzero value for $x \Delta s^{+}$for small values of $x$. This is a significant outcome and provides a valuable input for phenomenological studies.

Conclusions.-Results for the up, down, and strange quark helicity PDFs of the proton, within lattice QCD are presented for the first time using the quasi-PDFs approach. We compute matrix elements with nucleon states boosted to maximum momentum $P_{3}=1.24 \mathrm{GeV}$. We verify that the ground state matrix elements are well determined by using one- and two-state fits, confirming that $t_{s}=1.13 \mathrm{fm}$ is sufficiently large to suppress excited state contributions at this level of precision. The matrix elements are renormalized nonperturbatively, and matched to the light-cone PDFs using one-loop perturbation theory. For the flavor decomposition of the light quark PDFs we take into account, for the first time, both connected and disconnected diagrams and compute the totally disconnected strange PDF. The final results on $|x| \Delta q^{+}$and $|x| \Delta q^{-}$are shown in Fig. 3, and are compared with the global fits of the JAM and NNPDF Collaborations. We find a remarkable agreement for the case of $\Delta d^{+}$for all values of $x$ and for case of $\Delta u^{+}$for $x<0.6$. We also obtain $\Delta s^{+}$much more precise that the phenomenological determination and show that is clearly non-zero for small values of $x$. This work paves the way for a determination of these helicity PDFs using ensembles simulated with pion mass, which we plan to do in the near future.

In the near future, a number of sources of systematic uncertainties will be explored, using the particular ensemble, along the lines of the analysis of Ref. [34]. Another effect is the implementation of the mixing matching matrix between quark and gluon PDFs, which requires knowledge of the gluon matrix elements of nonlocal operators. Systematic uncertainties requiring more than one ensemble include discretization effects, volume effects, and pion mass dependence. We plan to assess a proper determination of all sources of systematic uncertainties for the individual flavor PDFs in the future. Once systematic uncertainties are addressed and quantified, lattice results can provide useful input in the global fits for the strange PDFs, as well as the individual light-quark PDFs. This calculation is a first step towards achieving this goal.

We would like to thank all members of ETMC for their constant and pleasant collaboration. We also thank N. Sato for providing the global fits data and J. Green for his comments. Finally, our thanks go to A. Scapellato for providing us the data at $0.43 \mathrm{GeV}$ for the connected matrix elements. M.C. acknowledges financial support by the U.S. Department of Energy Early Career Award under Grant No. DE-SC0020405. K. H. is supported by the Cyprus Research and Innovation Foundation under Grant No. POST-DOC/0718/0100. F. M. is supported by the European Joint Doctorate program STIMULATE of the European Unions Horizon 2020 research and innovation programme under Grant Agreement No. 765048. This research includes calculations carried out on HPC resources of the Cyprus Institute (the Cyclone and Cyclamen machines) and of Temple University supported in part by the National Science Foundation through major research instrumentation Grant No. 1625061 and by the U.S. Army Research Laboratory under Contract No. W911NF-16-20189. Computations for this work were carried out in part on facilities of the USQCD Collaboration, which are 
funded by the Office of Science of the U.S. Department of Energy. This research used resources of the Oak Ridge Leadership Computing Facility, which is a DOE Office of Science User Facility supported under Contract No. DEAC05-00OR22725. The gauge configurations have been generated by the Extended Twisted Mass Collaboration on the KNL (A2) Partition of Marconi at CINECA, through the Prace project Pra13_3304 "SIMPHYS."

[1] J. Gao, L. Harland-Lang, and J. Rojo, Phys. Rep. 742, 1 (2018).

[2] C. A. Aidala, S. D. Bass, D. Hasch, and G. K. Mallot, Rev. Mod. Phys. 85, 655 (2013).

[3] K. J. Eskola, P. Pakkinen, H. Paukkunen, and C. A. Salgado, Eur. Phys. J. C 77, 163 (2017).

[4] J. J. Ethier and E. R. Nocera, Annu. Rev. Nucl. Part. Sci. 70, 43 (2020).

[5] E. R. Nocera, R. D. Ball, S. Forte, G. Ridolfi, and J. Rojo (NNPDF Collaboration), Nucl. Phys. B887, 276 (2014).

[6] M. Constantinou et al., arXiv:2006.08636.

[7] J. J. Ethier, N. Sato, and W. Melnitchouk, Phys. Rev. Lett. 119, 132001 (2017).

[8] D. de Florian, G. A. Lucero, R. Sassot, M. Stratmann, and W. Vogelsang, Phys. Rev. D 100, 114027 (2019).

[9] E. R. Nocera, in 22nd International Symposium on Spin Physics (SPIN 2016) Urbana, IL, USA, 2016 (2017), arXiv:1702.05077.

[10] X. Ji, Phys. Rev. Lett. 110, 262002 (2013).

[11] X. Ji, Sci. China Phys. Mech. Astron. 57, 1407 (2014).

[12] X. Xiong, X. Ji, J.-H. Zhang, and Y. Zhao, Phys. Rev. D 90, 014051 (2014).

[13] J.-W. Chen, S. D. Cohen, X. Ji, H.-W. Lin, and J.-H. Zhang, Nucl. Phys. B911, 246 (2016).

[14] C. Alexandrou, K. Cichy, M. Constantinou, K. Hadjiyiannakou, K. Jansen, F. Steffens, and C. Wiese, Phys. Rev. D 96, 014513 (2017).

[15] R. A. Briceño, M. T. Hansen, and C. J. Monahan, Phys. Rev. D 96, 014502 (2017).

[16] M. Constantinou and H. Panagopoulos, Phys. Rev. D 96, 054506 (2017).

[17] C. Alexandrou, K. Cichy, M. Constantinou, K. Hadjiyiannakou, K. Jansen, H. Panagopoulos, and F. Steffens, Nucl. Phys. B923, 394 (2017).

[18] X. Ji, J.-H. Zhang, and Y. Zhao, Nucl. Phys. B924, 366 (2017).

[19] X. Ji, J.-H. Zhang, and Y. Zhao, Phys. Rev. Lett. 120, 112001 (2018).

[20] T. Ishikawa, Y.-Q. Ma, J.-W. Qiu, and S. Yoshida, Phys. Rev. D 96, 094019 (2017).

[21] J. Green, K. Jansen, and F. Steffens, Phys. Rev. Lett. 121, 022004 (2018).

[22] W. Wang, S. Zhao, and R. Zhu, Eur. Phys. J. C 78, 147 (2018).

[23] I. W. Stewart and Y. Zhao, Phys. Rev. D 97, 054512 (2018).

[24] T. Izubuchi, X. Ji, L. Jin, I. W. Stewart, and Y. Zhao, Phys. Rev. D 98, 056004 (2018).
[25] C. Alexandrou, K. Cichy, M. Constantinou, K. Jansen, A. Scapellato, and F. Steffens, Phys. Rev. Lett. 121, 112001 (2018).

[26] J.-H. Zhang, J.-W. Chen, L. Jin, H.-W. Lin, A. Schafer, and Y. Zhao, Phys. Rev. D 100, 034505 (2019).

[27] R. A. Briceno, J. V. Guerrero, M. T. Hansen, and C. J. Monahan, Phys. Rev. D 98, 014511 (2018).

[28] G. Spanoudes and H. Panagopoulos, Phys. Rev. D 98 , 014509 (2018).

[29] C. Alexandrou, K. Cichy, M. Constantinou, K. Jansen, A. Scapellato, and F. Steffens, Phys. Rev. D 98, 091503(R) (2018).

[30] Y.-S. Liu et al., Phys. Rev. D 101, 034020 (2020).

[31] A. Radyushkin, Phys. Lett. B 788, 380 (2019).

[32] J.-H. Zhang, X. Ji, A. Schafer, W. Wang, and S. Zhao, Phys. Rev. Lett. 122, 142001 (2019).

[33] Z.-Y. Li, Y.-Q. Ma, and J.-W. Qiu, Phys. Rev. Lett. 122, 062002 (2019).

[34] C. Alexandrou, K. Cichy, M. Constantinou, K. Hadjiyiannakou, K. Jansen, A. Scapellato, and F. Steffens, Phys. Rev. D 99, 114504 (2019).

[35] W. Wang, J.-H. Zhang, S. Zhao, and R. Zhu, Phys. Rev. D 100, 074509 (2019).

[36] J.-W. Chen, H.-W. Lin, and J.-H. Zhang, Nucl. Phys. B952, 114940 (2020).

[37] T. Izubuchi, L. Jin, C. Kallidonis, N. Karthik, S. Mukherjee, P. Petreczky, C. Shugert, and S. Syritsyn, Phys. Rev. D 100, 034516 (2019).

[38] K. Cichy, L. Del Debbio, and T. Giani, J. High Energy Phys. 10 (2019) 137.

[39] W. Wang, Y.-M. Wang, J. Xu, and S. Zhao, Phys. Rev. D 102, 011502(R) (2020).

[40] H.-D. Son, A. Tandogan, and M. V. Polyakov, Phys. Lett. B 808, 135665 (2020).

[41] J. R. Green, K. Jansen, and F. Steffens, Phys. Rev. D 101, 074509 (2020).

[42] Y. Chai et al., Phys. Rev. D 102, 014508 (2020).

[43] V. Braun, K. Chetyrkin, and B. Kniehl, J. High Energy Phys. 07 (2020) 161.

[44] S. Bhattacharya, K. Cichy, M. Constantinou, A. Metz, A. Scapellato, and F. Steffens, Phys. Rev. D 102, 111501 (2020).

[45] S. Bhattacharya, K. Cichy, M. Constantinou, A. Metz, A. Scapellato, and F. Steffens, Phys. Rev. D 102, 034005 (2020).

[46] S. Bhattacharya, K. Cichy, M. Constantinou, A. Metz, A. Scapellato, and F. Steffens, Phys. Rev. D 102, 114025 (2020).

[47] L.-B. Chen, W. Wang, and R. Zhu, Phys. Rev. D 102, 011503 (2020).

[48] L.-B. Chen, W. Wang, and R. Zhu, J. High Energy Phys. 10 (2020) 079.

[49] L.-B. Chen, W. Wang, and R. Zhu, arXiv:2006.14825 [Phys. Rev. Lett (to be published)].

[50] X. Ji, Y. Liu, A. Schfer, W. Wang, Y.-B. Yang, J.-H. Zhang, and Y. Zhao, Nucl. Phys. B964, 115311 (2021).

[51] J. Bringewatt, N. Sato, W. Melnitchouk, J.-W. Qiu, F. Steffens, and M. Constantinou, Phys. Rev. D 103, 016003 (2021).

[52] R. Zhang, H.-W. Lin, and B. Yoon, arXiv:2005.01124. 
[53] K.-F. Liu and S.-J. Dong, Phys. Rev. Lett. 72, 1790 (1994).

[54] W. Detmold and C. J. David Lin, Phys. Rev. D 73, 014501 (2006).

[55] V. Braun and D. Mueller, Eur. Phys. J. C 55, 349 (2008).

[56] G. S. Bali et al., Eur. Phys. J. C 78, 217 (2018).

[57] G. S. Bali, V. M. Braun, B. Glassle, M. Gockeler, M. Gruber, F. Hutzler, P. Korcyl, A. Schafer, P. Wein, and J.-H. Zhang, Phys. Rev. D 98, 094507 (2018).

[58] W. Detmold, I. Kanamori, C. J. D. Lin, S. Mondal, and Y. Zhao, Proc. Sci. LATTICE2018 (2018) 106 [arXiv:1810.12194].

[59] J. Liang, T. Draper, K.-F. Liu, A. Rothkopf, and Y.-B. Yang (XQCD Collaboration), Phys. Rev. D 101, 114503 (2020).

[60] Y.-Q. Ma and J.-W. Qiu, Phys. Rev. D 98, 074021 (2018).

[61] Y.-Q. Ma and J.-W. Qiu, Int. J. Mod. Phys. Conf. Ser. 37, 1560041 (2015).

[62] A. Radyushkin, Phys. Lett. B 767, 314 (2017).

[63] A. J. Chambers, R. Horsley, Y. Nakamura, H. Perlt, P. E. L. Rakow, G. Schierholz, A. Schiller, K. Somfleth, R. D. Young, and J. M. Zanotti, Phys. Rev. Lett. 118, 242001 (2017).

[64] A. V. Radyushkin, Phys. Rev. D 96, 034025 (2017).

[65] K. Orginos, A. Radyushkin, J. Karpie, and S. Zafeiropoulos, Phys. Rev. D 96, 094503 (2017).

[66] Y.-Q. Ma and J.-W. Qiu, Phys. Rev. Lett. 120, 022003 (2018).

[67] A. V. Radyushkin, Phys. Lett. B 781, 433 (2018).

[68] A. Radyushkin, Phys. Rev. D 98, 014019 (2018).

[69] J.-H. Zhang, J.-W. Chen, and C. Monahan, Phys. Rev. D 97, 074508 (2018).

[70] J. Karpie, K. Orginos, and S. Zafeiropoulos, J. High Energy Phys. 11 (2018) 178.

[71] R. S. Sufian, J. Karpie, C. Egerer, K. Orginos, J.-W. Qiu, and D. G. Richards, Phys. Rev. D 99, 074507 (2019).

[72] B. Jo, J. Karpie, K. Orginos, A. Radyushkin, D. Richards, and S. Zafeiropoulos, J. High Energy Phys. 12 (2019) 081.

[73] A. V. Radyushkin, Phys. Rev. D 100, 116011 (2019).

[74] B. Joo, J. Karpie, K. Orginos, A. V. Radyushkin, D. G. Richards, R. S. Sufian, and S. Zafeiropoulos, Phys. Rev. D 100, 114512 (2019).

[75] I. Balitsky, W. Morris, and A. Radyushkin, Phys. Lett. B 808, 135621 (2020).

[76] A. Radyushkin, Int. J. Mod. Phys. A 35, 2030002 (2020).

[77] R. S. Sufian, C. Egerer, J. Karpie, R. G. Edwards, B. Jo, Y.-Q. Ma, K. Orginos, J.-W. Qiu, and D. G. Richards, Phys. Rev. D 102, 054508 (2020).

[78] B. Jo, J. Karpie, K. Orginos, A. V. Radyushkin, D. G. Richards, and S. Zafeiropoulos, Phys. Rev. Lett. 125, 232003 (2020).

[79] M. Bhat, K. Cichy, M. Constantinou, and A. Scapellato, arXiv:2005.02102 [Phys. Rev. D (to be published)].

[80] K. Can et al., Phys. Rev. D 102, 114505 (2020).
[81] C. Alexandrou, K. Cichy, M. Constantinou, K. Hadjiyiannakou, K. Jansen, A. Scapellato, and F. Steffens, Phys. Rev. Lett. 125, 262001 (2020).

[82] K. Cichy and M. Constantinou, Adv. High Energy Phys. 2019, 1 (2019).

[83] X. Ji, Y.-S. Liu, Y. Liu, J.-H. Zhang, and Y. Zhao, arXiv:2004.03543.

[84] M. Constantinou, arXiv:2010.02445.

[85] C. Alexandrou, S. Bacchio, M. Constantinou, J. Finkenrath, K. Hadjiyiannakou, K. Jansen, G. Koutsou, and A. Vaquero Aviles-Casco, Phys. Rev. D 102, 054517 (2020).

[86] M. Constantinou, M. Hadjiantonis, H. Panagopoulos, and G. Spanoudes, Phys. Rev. D 94, 114513 (2016).

[87] J. C. Collins, Renormalization: An Introduction to Renormalization, the Renormalization Group and the Operator-Product Expansion, Cambridge Monographs on Mathematical Physics (Cambridge University Press, Cambridge, England, 1984).

[88] J. Karpie, K. Orginos, A. Rothkopf, and S. Zafeiropoulos, J. High Energy Phys. 04 (2019) 057.

[89] C. Alexandrou, G. Iannelli, K. Jansen, and F. Manigrasso (Extended Twisted Mass), Phys. Rev. D 102, 094508 (2020).

[90] C. Alexandrou et al., Phys. Rev. D 98, 054518 (2018).

[91] G. S. Bali, B. Lang, B. U. Musch, and A. Schafer, Phys. Rev. D 93, 094515 (2016).

[92] A. Abdel-Rehim, C. Alexandrou, M. Constantinou, J. Finkenrath, K. Hadjiyiannakou, K. Jansen, C. Kallidonis, G. Koutsou, A. V. Avils-Casco, and J. Volmer, Proc. Sci. LATTICE2016 (2016) 155 [arXiv:1611.03802].

[93] A. Stathopoulos, J. Laeuchli, and K. Orginos, arXiv: 1302.4018.

[94] A. Abdel-Rehim, C. Alexandrou, M. Constantinou, V. Drach, K. Hadjiyiannakou, K. Jansen, G. Koutsou, and A. Vaquero, Phys. Rev. D 89, 034501 (2014).

[95] C. Alexandrou, M. Constantinou, V. Drach, K. Hadjiyiannakou, K. Jansen, G. Koutsou, A. Strelchenko, and A. Vaquero, Comput. Phys. Commun. 185, 1370 (2014).

[96] C. Alexandrou, S. Bacchio, M. Constantinou, J. Finkenrath, K. Hadjiyiannakou, K. Jansen, G. Koutsou, H. Panagopoulos, and G. Spanoudes, Phys. Rev. D 101, 094513 (2020).

[97] C. Alexandrou, S. Bacchio, M. Constantinou, J. Finkenrath, K. Hadjiyiannakou, K. Jansen, and G. Koutsou, Phys. Rev. D 101, 031501 (2020).

[98] C. Alexandrou, S. Bacchio, M. Constantinou, J. Finkenrath, K. Hadjiyiannakou, K. Jansen, G. Koutsou, and A. V. Aviles-Casco, Phys. Rev. D 100, 014509 (2019).

[99] A. Buckley, J. Ferrando, S. Lloyd, K. Nordström, B. Page, M. Rüfenacht, M. Schönherr, and G. Watt, Eur. Phys. J. C 75, 132 (2015).

[100] C. Alexandrou, K. Cichy, M. Constantinou, J. R. Green, K. Hadjiyiannakou, K. Jansen, F. Manigrasso, A. Scapellato, and F. Steffens, arXiv:2011.00964. 